\section{Neuroleptic malignant syndrome due to risperidone misdiagnosed as status epilepticus}

\author{
Ali Ertug Arslankoylu, Meryem Ozlem \\ Kutuk, Cetin Okuyaz, Fevziye Toros \\ Mersin University Faculty of Medicine, \\ Department of Pediatrics, Pediatric \\ Intensive Care Unit, Mersin, Turkey
}

\begin{abstract}
Neuroleptic malignant syndrome (NMS) is a rare but potentially fatal disease characterized by fever, muscle rigidity, delirium and autonomic instability. Here we report a child, with NMS due to the risperidone misdiagnosed as status epilepticus. Nine year old boy, who had been under high dose risperidone treatment for 8 weeks, admitted to the emergency room because of the contractions (evaluated as status epilepticus) persisting for 7 hours. Since there was neuroleptic treatment in the past medical history and, unconsciousness, muscular rigidity, diaphoresis, hypertermi and, hypotension in physical examination, leucocytosis and elevated creatininphosphokinase levels in laboratory tests, the patient was evaluated as NMS and discharged without any complications. We reported this case to point out that; NMS may be misdiagnosed as status epilepticus in children when EEG monitoring is unavailable. When a child admitted to the emergency room because of suspicious convulsion neuroleptic drug use must surely be asked.
\end{abstract}

\section{Introduction}

Neuroleptic malignant syndrome (NMS) is a rare but potentially fatal disease characterized by fever, muscular rigidity, delirium and autonomic instability. ${ }^{1}$ Yearly prevalence of the disease in patients under neuroleptic treatment ranges between $0.02 \%$ and $2.4 \%$. Mortality rate of the patients without specific treatment is approximately $21 \% 0^{2,3}$ Mortality rate may be higher in patiens with misdiagnosis like our patient. The classic presentation of this syndrome has been most commonly associated with the typical neuroleptic drugs such as haloperidol. ${ }^{2}$

Risperidone is a new atypical neuroleptic agent. 5-HT2A antagonism partially protects against D2 antagonist-induced-neurologic side effects and, may improve negative symptoms and cognitive functioning via modulation of mesocortical DA activity. In addition, blockade of adrenoceptors may further increase prefrontal cortical activity and could enhance antipsychotic efficacy by modulations of mesolimbic DA activity. Unlike other atypical agents, risperidone does not differ from conventional agents in its dissociation contrast for D2 receptor; this feature perhaps accounts for the risk of EPS (extrapyramidal side effects) at high doses. ${ }^{4}$ However in large multicentre studies it was shown that risperidone had similar side effects like other typical neuroleptic agents. ${ }^{5}$ As far as we know the number of cases due to risperidone treatment in the literature is limited and most of the reported cases are adults. In most of these cases risperidone induces NMS after two weeks of treatment. ${ }^{6}$

Here we report a child with NMS due to risperidone treatment who was misdiagnosed as status epilepticus. The patient reported here met DSM-IV criteria for NMS.1

\section{Case Report}

Nine years old boy was admitted to the emergency department because of the convulsions persisting for seven hours. He had attention deficit hyperactivity disorder and he had been under risperidone $(2 \times 2 \mathrm{mg} /$ day $)$ treatment orally for 8 weeks because of moderate mental retardation and psychological disorder. Risperidone dose was four times more than usually recommended pediatric dosage. Additionally the patient had been suffering vomiting, tremor and fever for two weeks.

Physical examination in the emergency room revealed a body temperature of $39^{\circ} \mathrm{C}$, a respiratory rate of 10 breaths/min, and, a pulse rate of 158 beats/min. Blood pressure was too low that it couldn't be measured initially. The patient's general appearance was lethargic. There was muscular rigidity, tremor and, diaphoresis. He was dehydrated. Heart sounds were rhythmic and tachycardic. His respiration was weak and respiratory sounds were normal. Bilateral pupil reflexes were positive. Deep tendon reflexes were brisk and bilateral Babinski reflexes were positive. There were no meningeal irritation signs.

Initial laboratory analysis revealed a white blood cell count of 21580 cells $/ \mathrm{mm}^{3}$, with $2 \%$ band neutrophils, $62 \%$ neutrophils, $30 \%$ lymphocytes, 6\% monocytes. Serum electrolytes were in normal ranges included a sodium level of $136 \mathrm{mEq} / \mathrm{L}$; potassium, $5.4 \mathrm{mEq} / \mathrm{L}$; chloride $105 \mathrm{mEq} / \mathrm{L}$ and, calcium $8.2 \mathrm{mE} / \mathrm{L}$. The blood glucose was $316 \mathrm{mg} / \mathrm{dl}$. Initial venous blood gases showed a pH of 7.04; carbon dioxide tension $95 \mathrm{mmHg}$; bicarbonate $26 \mathrm{mEq} / \mathrm{L}$ and, base excess, $-5.8 \mathrm{mmol} / \mathrm{L}$. The serum creatinine phosphokinase (CPK) level was $1658 \mathrm{U} / \mathrm{L}$. Blood, urine and stool cultures were negative. Urinalysis, chest radiograph, cranial CT and
Correspondence: Ali Ertug Arslankoylu, Mersin University Faculty of Medicine, Department of Pediatrics, Pediatric Intensive Care Unit. 33079, Mersin, Turkey.

Tel. +90.324.3374300.1161 - Fax: +90.324 3374305 E-mail: aliertug@gmail.com

Key words: neuroleptic malignant syndrome, risperidone, status epilepticus.

Contributions: AEA, manuscript preparation supervision; MOK, data conception and design; $\mathrm{CO}$, FT, manuscript revision; AEA,MOK, C0, FT, final manuscript approval.

Received for publication: 27 May 2011. Accepted for publication: 21 June 2011.

This work is licensed under a Creative Commons Attribution NonCommercial 3.0 License (CC BYNC 3.0).

(C) Copyright A.E. Arslankoylu et al., 2011

Licensee PAGEPress, Italy

Pediatric Reports 2011; 3:e19

doi:10.4081/pr.2011.e19

EEG were unremarkable.

Although the tremors accompany muscular rigidity were mimicking convulsions and the patient was admitted with staus epilepticus diagnosis, the patient was not diagnosed as status epilepticus because there were neuroleptic treatment in his medical history, muscular rigidity, diaphoresis, hypertermi and hypotension in physical examination, leucocytosis and elevated CPK levels in laboratory tests. By the help of the sign and symptoms above, the patient was diagnosed as NMS by pediatric critical care intensivist, pediatric neurologist and pediatric psychiatrist. NMS diagnosis was confirmed with EEG and risperidone treatment was ceased.

In the emergency department the patient was entubated because of respiratory failure. Then he was admitted to the pediatric intensive care unit and mechanically ventilated. Intravenous diazepam infusion $(0.3 \mathrm{mg} / \mathrm{kg}$ hour) and carbidopa/levodopa were given for NMS treatment. In the first day of the admission to the intensive care unit the patient had hyperthermia resistant to the antipyretic treatment and his blood pressure was unstable. Central venous catheter was placed and hemodynamic parameters of the patient were regulated in normal ranges. In the second day of the admission to the intensive care unit the patient was extubated. All the clinical and laboratory signs and symptoms of the patient improved except elevated creatinin phosphokinase levels which turned back to normal values on the fifth day of the admission. Then he was discharged from the hospital without complications. 
with dopamine.

Previously it was thought that NMS was only associated with the use of classical neurolep-

Here we report a patient with NMS who was misdiagnosed as status epilepticus before admission to our hospital. Our patient was diagnosed as NMS due to presence of mentioned criteria of NMS. ${ }^{1}$ Mortality rate of NMS in the patients without specific treatment is approximately $21 \% .2,3$ Mortality rate may be higher in patiens with misdiagnosis. NMS signs and symptoms may be difficult to differentiate from status epilepticus by inexperienced physicians when EEG monitoring is unavailable. As mentioned before, our patient was diagnosed as status epilepticus before he was admitted to our hospital and he had been tried to be treated as status epilepticus at the beginning.

The clinical signs and symptoms of our patient emerged in the second week of the risperidone treatment. As it was pointed out before NMS may occur in anytime during neuroleptic treatment. ${ }^{6}$

First step of the NMS treatment is to cease antipsychotic drugs. After we diagnosed NMS we discontinued the risperidone treatment. Cooler blankets and antipyretics for hyperthermia and supportive treatment including fluid electrolyte therapy must be supplied. In medical treatment in order to reduce muscular rigidity dopamine agonists are used. Similarly we used dopamine agonists for our patient. Additionally dantrolene may be used alone or tics. However there are some NMS cases due to atypical neuroleptics in the literature. ${ }^{7}$ As far as we know in the literature only two NMS cases due to risperidone treatment were reported in children. ${ }^{8,9}$ We think that atypical neuroleptics will be extensively used in children in the future and the clinicians especially the pediatricians must be familiar to NMS and its differential diagnosis.

We report this case in order to underline that neuroleptic malignant syndrome may be misdiagnosed as status epilepticus in children when EEG monitoring is unavailable. When a child is admitted to the emergency department because of unconsciouesness muscular rigidity, diaphoresis, hypertermi and hypotension; neuroleptic drug use must surely be asked. Furthermore this is the third neuroleptic malignant syndrome case due to risperidone treatment in children. Early diagnosis and proper treatment of NMS may decrease the mortality rates significantly.

\section{References}

1. American Psychiatric Association. Diagnostic and statistical manual of mental disorders, Revised fourth edition. Washington, DC: American Psychiatric Press; 2000.
2. Leibold J, Patel V, Hasan RA. Neuroleptic malignant syndrome associated with ziprasidone in an adolescent. Clin Ther 2004;26:1105-8.

3. Steingard R, Khan A, Gonzales A, Herzog D. Neuroleptic malignant syndrome: review of experience with children and adolescents. J Child Adolesc Psychopharmacol 1992;2:183-98.

4. Gertz HJ, Schmidt LG. Low melatonin content of substantia nigra in a case of neuroleptic malignant syndrome. Pharmacopsychiatry 1991;24:93-5.

5. Ballard C, Grace J, Mckeith J, Holmes C. Neuroleptic sensitivity in dementia with Lewy bodies and Alzheimer's disease. Lancet 1998;351:1032.

6. Caroff SN, Mann SC. Neuroleptic malignant syndrome. Med Clin North Am 1993; 77:185-202.

7. Srivastava A, Borkar HA, Chandak S. Olanzapine-induced neuroleptic malignant syndrome in a patient with paranoid schizophrenia. Psychiatry Clin Neurosci 2009;63:119-21.

8. Chungh DS, Kim BN, Cho SC. Neuroleptic malignant syndrome due to three atypical antipsychotics in a child. J Psychopharmacol 2005;19:422-5.

9. Vurucu S, Congologlu A, Altun D, et al. Neuroleptic malignant syndrome due to risperidone treatment in a child with Joubert syndrome J Natl Med Assoc 2009; 101:273-5. 
